\title{
THE DIGITAL SYSTEM ARTEMIS FOR REAL-TIME PROCESSING OF RADIO TRANSIENT EMISSIONS IN THE SOLAR CORONA
}

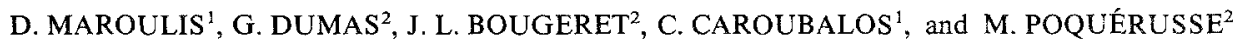

(Received 29 December, 1991; in revised form 11 August, 1992)

\begin{abstract}
We present the real-time digital data processing system named 'ARTEMIS' that was developed and constructed by the Space Research Department (DESPA) of Paris-Meudon Observatory to digitize, calibrate, format, date, process, compress, and archive in real time signals from multichannel receivers. This system is controlled by a multiprocessor computer based on Motorola MC 68010/68020 processors; it permits the automatic, routine recording of 128 parallel channels at a rate up to 300 samples per second and per channel with a 12-bit accuracy ( 4096 levels of intensity); it is used to process and record the 120 channels of a multichannel solar radiospectrograph in the frequency range $110-469 \mathrm{MHz}$; the remaining 8 channels are used for a scanning spectrograph in the frequency range $30-80 \mathrm{MHz}$ and a two-dimensional multicorrelator interferometer at $75.5 \mathrm{MHz}$. The large quantity of raw data is reduced in real-time from about 1.3 Gbytes to about 40 Mbytes per day by the use of an original algorithm for real-time data compression. It is expected that this new facility will allow us to build a very large data base of digitized and accurately calibrated solar events, in order to achieve statistical measurements over long periods of time.
\end{abstract}

\section{Introduction}

The dynamic spectrum of solar radio bursts is fundamental to the analysis of the solar corona for many reasons. The observed radio frequency is directly related to the electron density at the source, so that decreasing frequency is interpreted as increasing distance from the Sun. For instance, the frequency range from 469 to $30 \mathrm{MHz}$ covers the distance range from the top of the transition region up to about 2.5 solar radii $\left(R_{\odot}\right)$ from the center of the Sun. Solar radiospectrographs frequently have resolutions of the order of $1 \mathrm{MHz}$, which corresponds to an altitude variation in the corona of about $10^{-3} R_{\odot}$; this corresponds to a radial resolution two orders of magnitude better than the (transverse) resolution of interferometers (radioheliographs). The dynamic spectrum represents a synthetic overall measurement of solar radio phenomena which is not distorted by propagation effects and provides a physical signature of many solar events: electron streams, shock waves, trapped particles, etc. However, meter and decimeter observations require a very high resolution, both in time $(\approx 1 \mathrm{~ms}$ in the decimeter range, $10-100 \mathrm{~ms}$ in the meter range) and in frequency $(\Delta f / f \approx \mathrm{a}$ few $\%)$, and a high accuracy (a few $0.01 \mathrm{~dB}$ ) in a large dynamic range (greater than $40 \mathrm{~dB}$ above the background). Accurate calibrations in intensity and frequency are also required.

Although there exist many spectrographs that monitor solar radio activity around the world, few utilize the most recent techniques of real-time data processing. The scan-

${ }^{1}$ Department of Informatics, Division of Communications and Signal Processing, Ktiria TYPA, University of Athens, GR-15771, Ilissia, Greece.

${ }^{2}$ Space Research Department (DESPA), URA CNRS 264, Paris-Meudon Observatory, F-92195 Meudon, Cedex, France. 
ning spectrograph of Zürich (Perrenoud, 1982) is computer controlled. It uses a burst detector algorithm. It allows the recording of 2000 measurements $\mathrm{s}^{-1}$ of intensity and circular polarization, with an 8-bit accuracy (256 levels of intensity). The microwave spectrograph of Bern (Stähli and Fuhrer, 1987) is controlled by a multiprocessor computer system (Motorola MC 68000). It allows the recording of the intensity and polarization of 32 channels at a rate up to about 200000 measurements $\mathrm{s}^{-1}$, with a 12-bit accuracy (4096 levels of intensity). At the maximum data rate, the data storage unit ( 84 Mbytes hard disk) permits a total of $3 \mathrm{~min}$ of observation. An on-line burst detection algorithm is used to cover longer periods of time.

The Solar Radio Spectrograph of the Space Research Department (DESPA) of Paris-Meudon Observatory, has been operated daily at the Nançay Radio Astronomy Station since 1979 (Dumas, Caroubalos, and Bougeret, 1982). It offers high resolution in time and intensity. Until March 1988 its outputs were digitized on 32 channels at

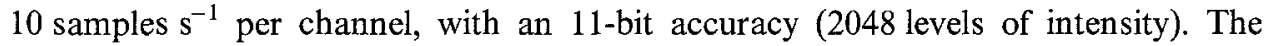
amount of data was later reduced by a factor $\approx 10$, by selecting and extracting active periods from the raw data tapes. A catalog of all archived periods is available on request (Crépel et al., 1989) and can also be interactively accessed from any computer which can access the European SPAN (International DECNet) network.

However, this system (used through March 1988) required many manipulations and a sustained effort from experienced personnel in order to select, extract and calibrate active periods. It was also drastically reducing the capabilities of the receiver, especially in time and frequency resolution. This led us to design and develop a much more powerful digital system that was named ARTEMIS ('Appareil de Routine pour le Traitement et l'Enregistrement Magnétique de l'Information Spectrale').

This paper is devoted to the description of this new system, whose main scientific objectives are to provide ancillary data to the WAVES investigation on the WIND spacecraft (to be launched in 1994), to analyze fine structures of solar events in the meter and decimeter bands, and to build up a large, accurate data base of digitized solar radio events, part of which will be integrated into the current international data bases.

The fulfilment of these objectives requires digital recordings of the solar radio dynamic spectrum in as large a frequency band as possible on a daily basis (routine observations) with high time, frequency and intensity resolution and an accurate calibration system. A major difficulty is that the ARTEMIS system yields more than 1 Gbyte of raw data per day. Hence, the data have to be compressed in real time by a large factor (10 to 100 ) without losing too much astrophysical information.

We present an overview of the scientific instruments in Section 2. We describe the hardware and the software that were developed for this new digital system in Sections 3 and 4 , and we give a few examples of the results in Section 5.

\section{The Observing Instruments}

Figure 1 shows the overall facilities which provide ground-based support to the spaceborne radio instruments of DESPA. 


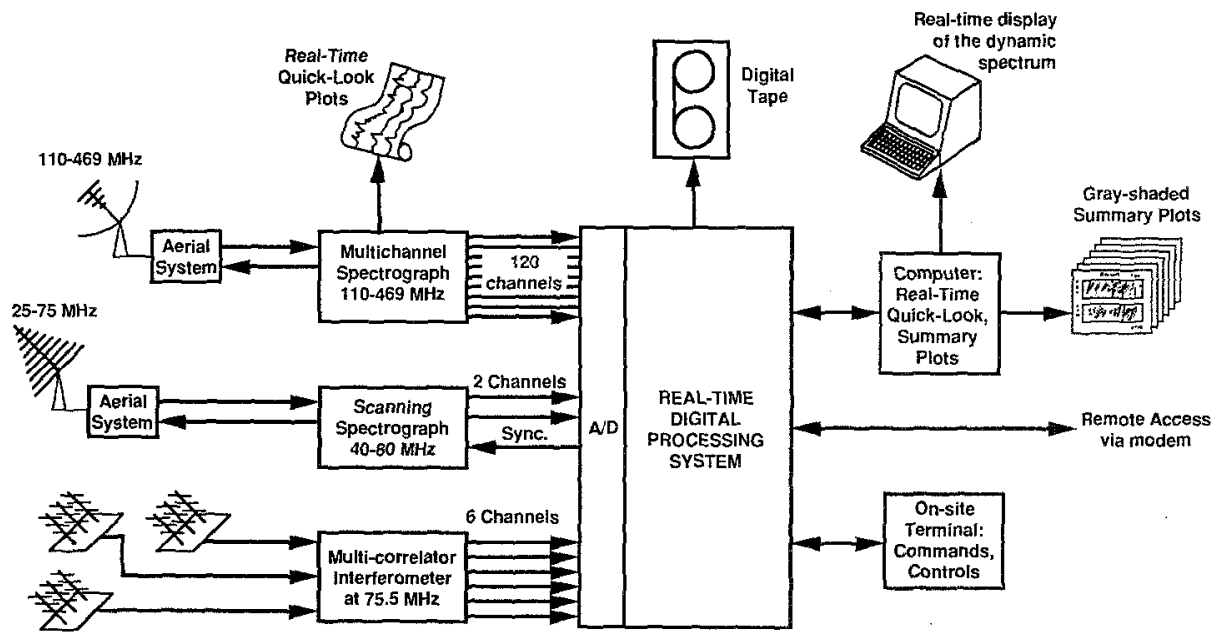

$75.5 \mathrm{MHz}$ Array

Fig. 1. Solar radio instruments of DESPA operated along with the ARTEMIS real-time digital processing system.

The Multichannel Spectrograph $(110-469 \mathrm{MHz})$

This was designed and constructed by DESPA and covers the frequency range $110-$ $469 \mathrm{MHz}$ (Dumas, Caroubalos, and Bougeret, 1982). It uses a crossed log-periodic antenna mounted on a $6-\mathrm{m}$ dish tracking the Sun from sunrise to sunset (gain $\approx 20 \mathrm{~dB}$ ). Its 120 channels yield a sensitivity $\sqrt{120} \approx 11$ times higher than a scanning instrument with an equivalent number of channels. It permits accurate frequency measurements and an excellent calibration, both absolute and relative for all channels. The problem of intermodulation in a large overall bandwidth is solved by distributing amplification among different stages of a binary 'tree' of power and band splitters, i.e., by avoiding high gains over wide bandwidths. The output of the receiver is proportional to the logarithm of the antenna temperature, which increases the dynamic range of the instrument $(\approx 50 \mathrm{~dB}$ ) without gain switching. A matrix of mixers (see Figure 1 in Dumas, Caroubalos, and Bougeret, 1982) is used in order to allow the selection of any frequency within the range $110-469 \mathrm{MHz}$ (360 possibilities), up to a total number of 120 filters of $1 \mathrm{MHz}$. IF passband filters are also available, which provide another 80 channels for high-frequency resolution analysis $(200 \mathrm{kHz}$ bandwidth). A logarithmic distribution of frequencies is used in the default operating mode (a feature well adapted to the description of solar events). Daily observations start with a calibration procedure during which the antenna is replaced by a sequence of 65 noise levels: 1 'absolute' level, accurately known in equivalent temperature and 64 'relative' levels spaced $1 \mathrm{~dB}$ apart and covering the whole dynamic range of the receiver (see Figure 6, top left).

\section{The Scanning Spectrograph (30-80 $\mathrm{MHz}$ )}

This utilizes a log-periodic antenna system whose gain is $\approx 10 \mathrm{~dB}$ (du Chaffaut, 1971). The spectrum analyzer was specially designed and constructed by the ARTEMIS team, at DESPA. It supports two special features: 
- a logarithmic expansion of frequency over time, so that the resulting frequency scale is logarithmic;

- an exact down ratio of scanning rate to the A/D sampling rate in order to synchronize the frequency scanning ( 5 scans per second) and permit the digital recording by regularly sampling the frequency band from 30 to $80 \mathrm{MHz}$.

Two digital channels are used: one for the intensities and the other for the frequency (synchronization signal).

\section{The Triangular Multicorrelator Interferometer at $75.5 \mathrm{MHz}$}

Three identical antenna systems form an isoceles triangle with an E-W base of $240 \mathrm{~m}$ and a N-S height of the same length. Each antenna system consists of three Yagi (1928) antennas tuned to $75.5 \mathrm{MHz}$, mounted on a $7 \mathrm{~m} \times 3 \mathrm{~m}$ flat reflector giving a gain of $14 \mathrm{~dB}$; it is steerable in the meridian plane only, and allows measurements \pm 1.5 hours around the local noon. After correction for ionospheric effects, two-dimensional positions on the Sun are obtained with an accuracy of $\pm 2^{\prime}$ (Daigne, Caroubalos, and Bougeret, 1975).

The 6 outputs (sine and cosine of the phase difference between each pair of antennas) are digitized and processed by ARTEMIS.

\section{Data Displays}

Until May 1991, the dynamic spectrum was displayed continuously on a $35 \mathrm{~mm}$ film running about 8 hours every day. The frequency bands were:

- since December 1978: 469-150 MHz,

- since January 1986: 469-110 MHz,

- since July 1986: $\quad 469-110 \mathrm{MHz}$ and $80-40 \mathrm{MHz}$.

Presently, digital data only is being used except for 6 control channels recorded on paper charts. The dynamic spectrum over the last 50 minutes is permanently displayed on a graphics monitor; it allows us to identify the activity in real time and to check the good working order of the equipment. Summary gray-scale plots are produced automatically at the end of each day on a laser printer (see below and Figure 4).

\section{The ARTEMIS Real Time Data Processing System (Hardware)}

\section{Overall Requirements (see Figure 2 for a summary)}

The main tasks to be performed routinely in real time have the following characteristics:

- acquisition of 128 analog channels, including 120 channels from the Multichannel Spectrograph, two channels from the Scanning Spectrograph, and 6 channels from the Triangular Multicorrelator Interferometer, with a possible extension to 256 channels;

- rates up to at least 100 data points $\mathrm{s}^{-1}$ and per channel (integration time $\approx 20 \mathrm{~ms}$, in order to comply with the sampling theorem), with a possible extension to $\approx 300$ data points $\mathrm{s}^{-1}$;

- 12 bit digitization (4096 levels of intensity); 


\section{ARTEMIS: Functional Diagram}

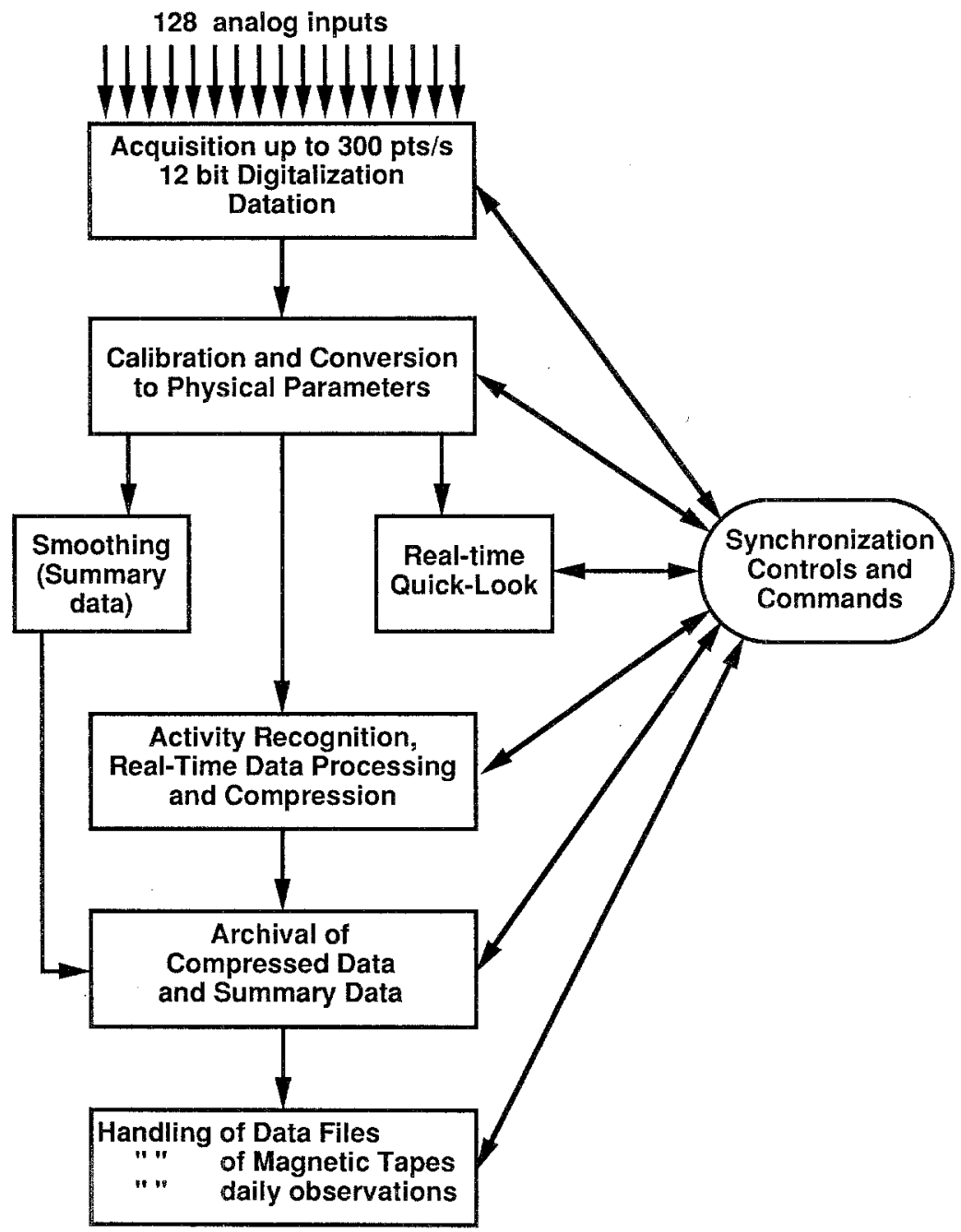

Fig. 2. Synoptic view of the main functions of the ARTEMIS digital processing system.

- automatic, absolute time marking (UT) with an accuracy better than $0.01 \mathrm{~s}$;

- calibration and conversion to physical parameters (antenna temperature);

- data compression, preserving details close to the instrument resolution (in time, frequency, and intensity);

- creation of a file of compressed data (on magnetic tape);

- creation of a data management file indicating the periods retained by the compression algorithm (tape);

- creation of a summary data file ( $5 \mathrm{~s}$ averages) (tape);

- creation of standard header and trailer files (tape); 
- real-time monitoring of the dynamic spectrum and of the status of the instrument;

- daily plotting of summary dynamic spectra.

All these tasks must be performed automatically at the required hours every day, so that at the observing station itself we instantly produce data in an archivable format that can be directly used for scientific exploitation. In addition, the hardware as well as the software must be reliable, simple, versatile, modular, and extendable.

\section{System Architecture}

The system is based on a multiprocessor architecture. Different tasks are concurrently executed in real-time by four Central Processing Units (CPU) on two VME buses. Practically, the overall digital system consists of two sub-systems connected by high data flow links (Figure 3):

- the Front End System, used for acquisition, digitization, formatting, time marking, conversion to antenna temperature, generation of summary data (all in real time);

- the Main System, used for the management of observations (mode, period, calibration), data compression, other real-time tasks defined by the user (additional compression, pattern recognition, etc.), sending the data to the tape drive, and also for various off-line tasks (file management, tape read/write).

This architecture isolates the different functions and makes the data acquisition independent from the real-time data processing. This preserves the computing power of the Main System for the execution of elaborate algorithms, and permits the acquisition to run at the full rate (up to 300 samples per second for each of the 128 channels).

\section{The Front End System (Figure 3, module 1)}

The VME bus of the Front End System supports:

- a motherboard with a Motorola MC 68010 CPU and 512 kbytes of DRAM (double access memory);

- a 1-Mbyte memory extension used for the real-time calibration of the data (conversion tables);

- two cards accepting 64 analog inputs each (0-5 V), multiplexing the inputs and converting into 12-bit digital data (XYCOM XVME 560);

- a bus controller with two serial input/outputs; the timing of the acquisition scans and the Universal Time and date are provided by a micro-processor-controlled radio clock which itself uses signals encoded in phase modulation on the 'France-Inter' AM radio broadcast;

- a communication module which includes another MC 68010 CPU, with one DMA (Direct Memory Access) controller (with four DMA channels) and 3 serial communication controllers (each with two serial ports).

The Main System (Figure 3, module 2)

The VME bus of the Main System supports:

- a motherboard with a Motorola MC $68020 \mathrm{CPU}$, a mathematics co-processor MC 68881, and 1 Mbyte of DRAM (double access memory); 


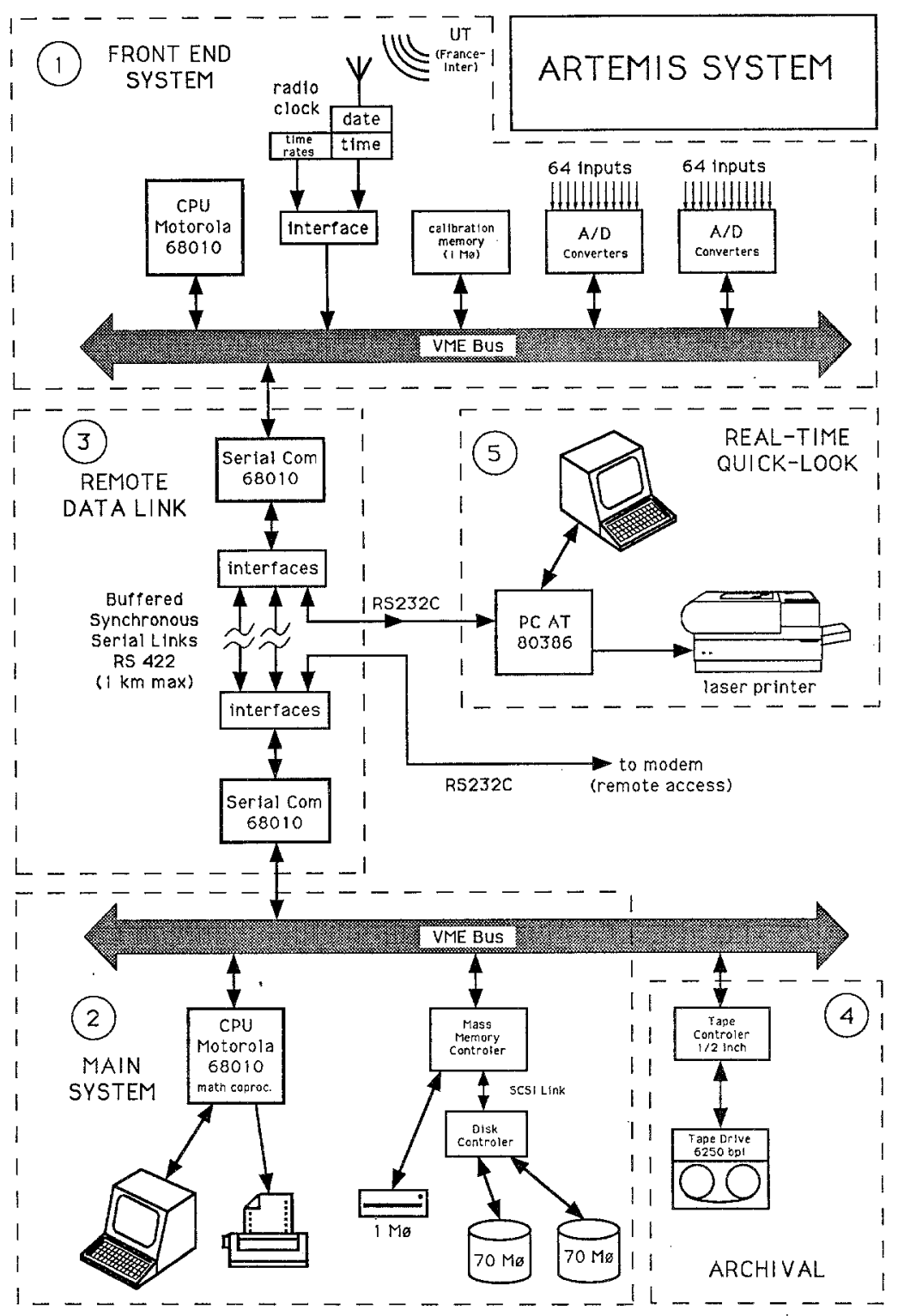

Fig. 3. Architecture of the ARTEMIS digital system. Tasks executed by different modules are described in the text.

- a bus controller which supports a floppy disk drive and two 70 Mbyte hard disks;

- a controller for a high density (6250 bpi) $\frac{1}{2} "$ magnetic tape (VTAPE 3209 of Interphase);

- a communication module which includes a MC 68010 CPU (similar to the communication module of the Front End System); it also helps to monitor the status of the instrument (screen display of the summary data and status parameters) and permits remote access to the Main System through a modem. 
The Data Link between the Front End System and the Main System (Figure 3, module 3)

The link between the two systems uses two intelligent serial communication modules. The data can be transferred from the Front End System to the Main System in a synchronous mode at a rate up to $1.5 \mathrm{Mbit} \mathrm{s}^{-1}$ (RS 422 standard link which works over distances up to $1 \mathrm{~km}$ ). Other links (RS 232 asynchronous mode) are used to pass commands and/or replies both ways from one system to the other and to communicate with other systems (real-time quick-look, remote access via modem, etc.).

Tape Drive (Figure 3, module 4)

The data are archived on $\frac{1}{2}^{\prime \prime}, 9$ track, high density (6250 bpi) magnetic tapes.

Real-Time, Quick-Look, Gray-Level Dynamic Spectrum (Figure 3, module 5)

Averages over $5 \mathrm{~s}$, calculated by the Front End System for each channel are sent by the communication module to a personal computer (PC AST 386SX/16) which drives a laser printer (Hewlett Packard LaserJet III). The PC processes the data, displays the spectrum on the monitor, and produces summary plots in the form of gray-shaded dynamic spectra (Figure 4). These plots can still be produced when the Main System is halted; they are used to monitor solar activity and for quick spectral identification.

\section{Software Development for the ARTEMIS System}

All software, including the communication drivers, have been developed by using the Motorola provided tools: VERSADOS operating system and specific debuggers.

The software for the Front End System and the communication drivers was written in assembly language and is not executed under the operating system, in order to optimize the speed. On the other hand, the Main System is operated under the VERSADOS operating system (real-time, multi-task, multi-user operating system). Many programs which run on this system (in real-time or off-line) are written in high level languages (FORTRAN 77, C).

\section{Calibration Table}

During a calibration sequence, the data are recorded on the hard disk. Then, using the 65 successive output levels, the Main System computes the antenna temperatures corresponding to all the 4096 intensity levels, for all the 120 channels, separately. Finally, this 1 Mbyte 'calibration table' is transferred through the synchronous serial communication link to the Front End System, where it is loaded in memory until the next calibration sequence.

\section{Acquisition, Calibration, and Pre-Processing}

The CPU of the Front End System drives the data acquisition and the analog-to-digital conversion according to parameters defined by the user. The default acquisition rate 


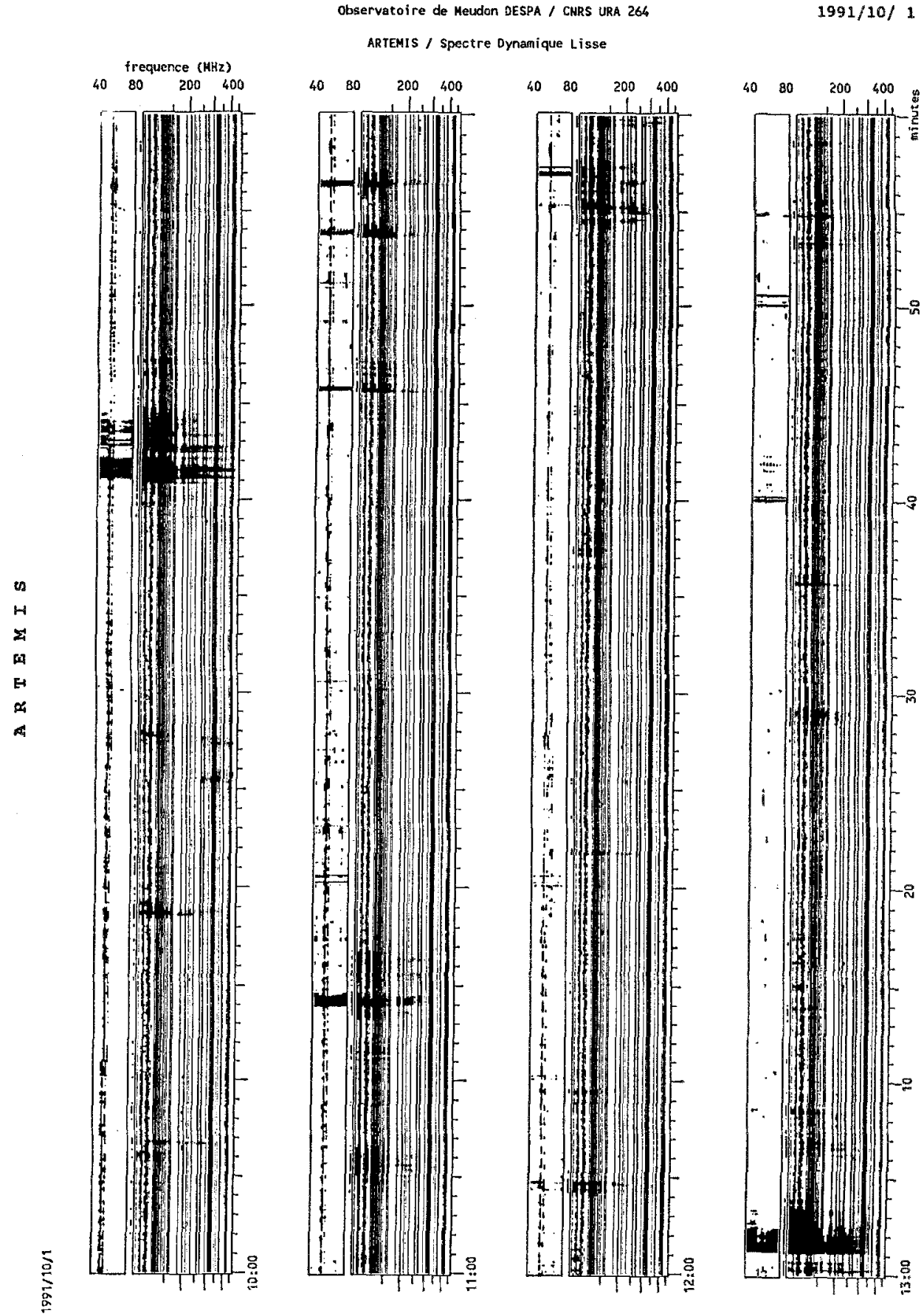

Fig. 4. Example of quick look. Total intensity and its derivative are printed every day on a total of 6 pages. 
is 100 data points $\mathrm{s}^{-1}$ for each channel. The Front End System replaces every data point by the antenna temperature read in the calibration table for the corresponding digitized voltage and channel. This real-time data calibration by 'transcoding' is extremely fast. The data are then buffered by 126 kbyte 'pages' consisting of 500 consecutive scans $(5 \mathrm{~s})$, plus a header containing date and time information and instrument parameters. The same processor also averages the calibrated data over every page $(0.2 \mathrm{~Hz})$ and sends a signal to the CPU of the communication card that gets the Universal Time from the radio clock to better than $0.01 \mathrm{~s}$.

\section{Communication Software}

The driver of the synchronous serial data transfer is executed by the communication CPU (Front End side). It gets the 126-kbyte page in a mode synchronized with the main CPU of the Front End System, and emits it on the serial port, with handshake, at a speed comprised between $300 \mathrm{kbit} \mathrm{s}^{-1}$ and $1.5 \mathrm{Mbit} \mathrm{s}^{-1}$, depending on the acquisition rate. Then, the driver on the reception or Main System side gets this page and delivers it to the Main System (in flip-flop). The emission and reception of the data frames are made by using the direct memory access channels (DMA).

Other asynchronous communication drivers have been developed that allow the user of the Main System to dialog with the Front End System, to send data to the quicklook plotting devices, or to access the Main System via a modem.

\section{Data Compression}

At an acquisition rate of 100 samples $\mathrm{s}^{-1}$, the raw data flow from the 128 channels is $200 \mathrm{kbits} \mathrm{s}^{-1}$, which would result in about 12 standard 6250-bpi magnetic tapes per day. In order to reduce this amount of data by a large factor without losing too much astrophysical information, we have developed a compression algorithm which restricts recording to periods with solar activity. The principle of this algorithm was described in detail elsewhere (Dumas, Caroubalos, and Bougeret, 1982).

The data set corresponding to the 500 consecutive points of a specific channel inside a 'page' is called a 'sentence'. Given the channel, a data point can be referenced by $x_{i, j}$, where $i$ and $j$ are, respectively, the index of the point within a sentence and the index of the sentence. Let $m_{j}$ be the arithmetic mean of the $N$ data points in the sentence $j$. If the quantity $\mu=\Sigma_{i=1}^{i=N}\left|x_{i, j}-m_{j-1}\right|$ computed in the $j$ th sentence is larger (smaller) than a given threshold $\sigma$, the channel is considered as 'active' (inactive) over that sentence.

This test is performed on a number of 'master channels'. A page is considered to be 'active' if at least one of the master channels is. The system retains or rejects pages as a whole according to the following rule: a page is retained if it is active or if it immediately precedes or follows an active page. The average $m_{j}$ over a page of all the 128 channels are always recorded, whether the page is retained or not.

We first tested the algorithm on the VAX computer of DESPA, using records of 
various types of solar events recorded at a high rate without any compression to obtain the best trade-off between compression ratio and loss of information. An example is given in Figure 5, which shows the results of tests made on eight different channels for three different thresholds. The algorithm, written in assembly language, was then implemented on the ARTEMIS system (Front End) to be executed in real-time.

This compression algorithm has been used successfully since September 1989. It is well adapted to the sporadic nature of solar radio activity and preserves the smallest details in time, frequency, and intensity. Moreover, most isolated, short duration interference is eliminated. In spite of the high level of activity (closeness to the solar

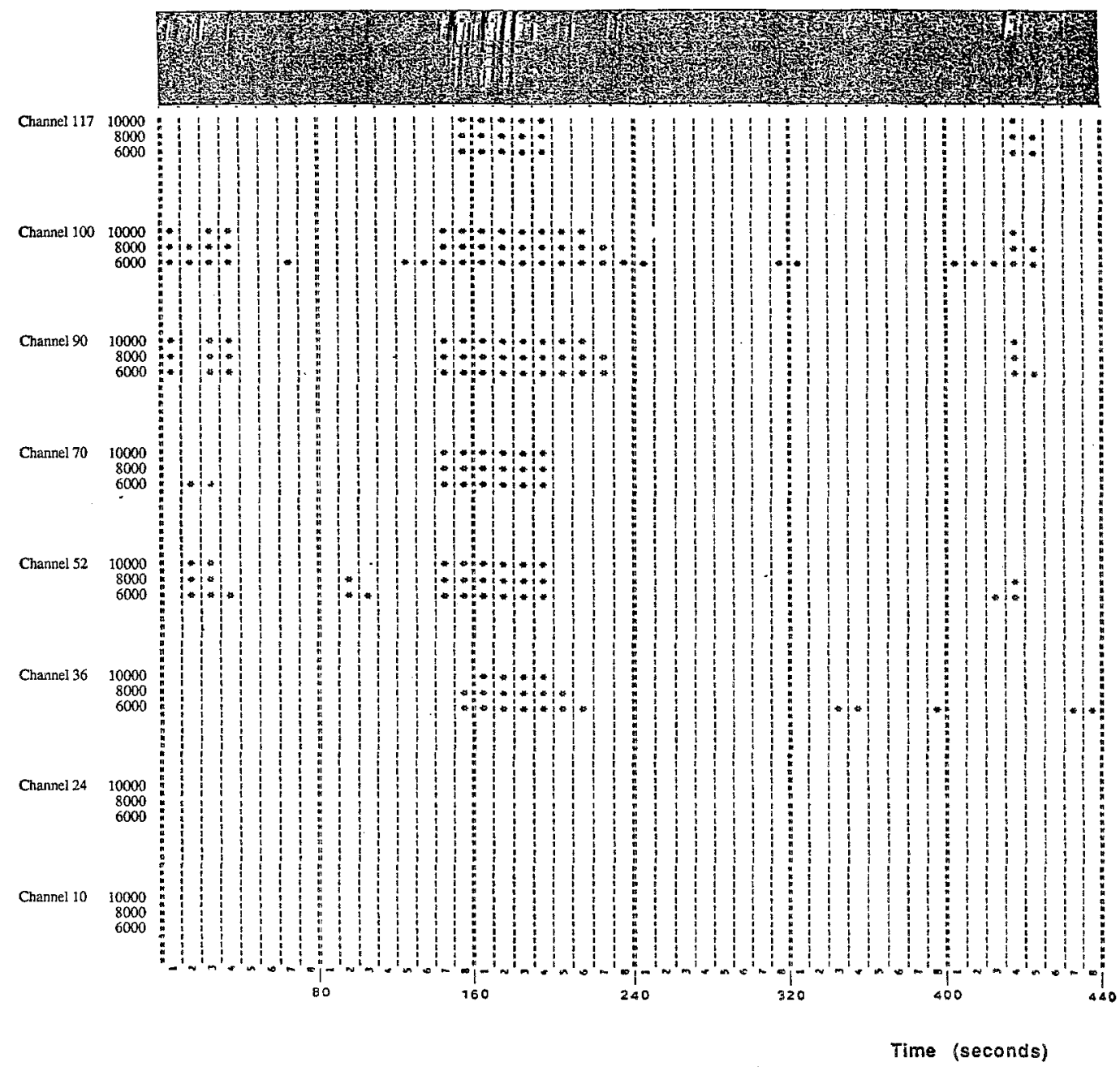

Fig. 5. Test of the decision threshold of the data compression algorithm. The band at the top displays the dynamic spectrum obtained without any compression. Vertical bars separate 'sentences' with 5-s duration. The results are presented for eight different frequencies. The asterisks show when the given thresholds are exceeded, corresponding to periods that the algorithm retains. 


\section{ARTEMIS 19911102 ACTIVE DATA $(100 \mathrm{~Hz})$}
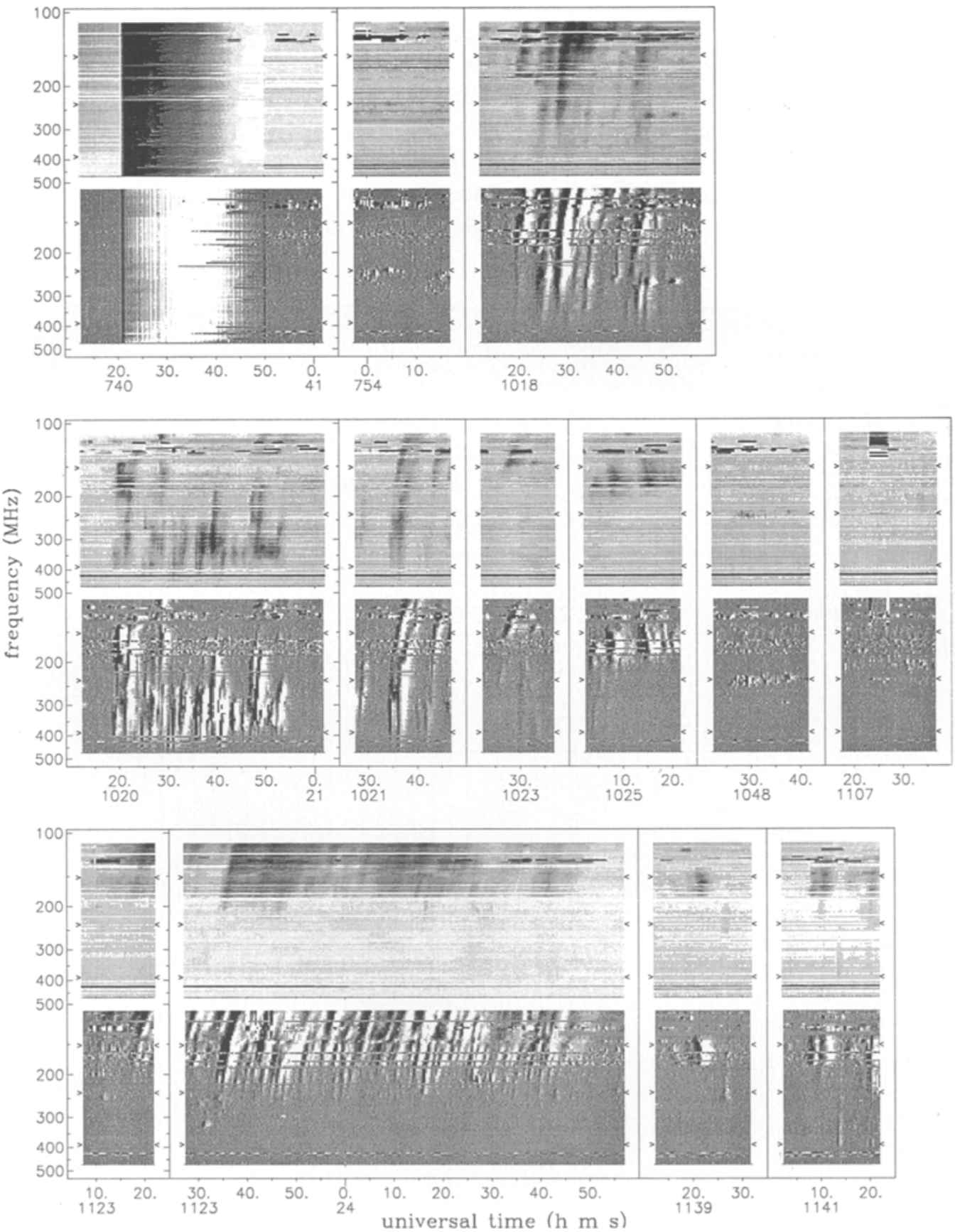

Fig. 6. Plot in standard mode (default parameters) of consecutive 'active' periods retained by the compression algorithm. Within each view (rectangular frame) the top panel is the intensity (gray-shaded scale automatically chosen among three pre-defined ranges), and the bottom panel is the time derivative (constant 
maximum), this algorithm has resulted in an average of two magnetic tapes per week (120 Mbytes per tape), which corresponds to an average compression ratio of about 40 .

\section{Magnetic Tape Structure}

The Main System manages the generation of the magnetic tapes according to a welldefined format. A tape contains five different kinds of files. It starts with:

- a header file in ASCII, where, among general parameters, the observer can enter a text of his choice.

The core of a tape consists of a succession of daily triplets of binary files. A triplet corresponds to a continuous observation period and consists of:

- an 'active data' file, containing all the pages of $100 \mathrm{~Hz}$ data selected by the compression algorithm;

- a management file indicating for every 5-s page whether it was selected or not;

- a 'smoothed data' file, containing the $0.2 \mathrm{~Hz}$ data over the whole observation period; this file is formatted in pages in the same way as the 'active data' file.

A magnetic tape ends with:

- a trailer file in ASCII, giving the dates of all the observation periods, the corresponding amounts of data and other summary parameters.

\section{ARTEMIS Software Package for Data Visualization and Reduction}

We maintain a catalog of all the periods archived on tape. It can be accessed interactively using a specialized routine from any computer connected to the European SPAN (International DECNet) network. Our 'active files' are made up of a succession of many short sequences of continuous data, with random time jumps between sequences. We developed a number of Fortran modules to deal with this and all kinds of file and format peculiarities. These elementary modules are basic tools for the development of higher level routines oriented towards the automatic treatment of large quantities of data.

The first-high level routine plots gray-level dynamic spectra on a laser printer, with automatic or customized page setting. Files (active or smoothed data), time periods and scales (in time, frequency, and intensity) are chosen at will. The user can also choose to subtract the background level (separately for each channel), or to plot the time derivative (enhancing low-contrast fine structures). To make it user-friendly, despite its numerous definable parameters, the program has predefined standard modes. In addition, the different parameters can be reached quickly through an interactive tree branching process in which the user tells the program what to do with sub-groups of parameters: take previous, standard, automatic, or user-defined values. Figures 6, 7, and 8 were all plotted with this program.

gray-shade scale). The derivative is best suited to detect small amplitude fluctuations. The program takes care of the page setting automatically; notice that it avoids cutting off continuous data periods (as long as they fit in the width of the page). The first view shows a calibration sequence. The other views mainly show type III groups. (The upper line on the time axis gives seconds; the lower line gives hours and minutes.) 
We are also developing routines within the commercial software 'IDL' to perform a number of tasks such as image processing, 1-D or 2-D Fourier analysis, convolutions, automatic measurements, etc.

\section{Examples of Observations}

Figure 6 is the first sheet of several plotted in a routine (standard) mode for a typical day. Within each frame, the top panel shows the intensity and the lower panel its time derivative (positive derivatives are dark and negative derivatives clear).

The first period retained by the compression system was the calibration sequence. Then the compression system kept groups of type III bursts, spike bursts and reversedrift bursts. A few periods have been kept due to weak activity or interference. In this standard plotting mode, the gray level scale for the intensity panels is chosen automatically for each frame separately, depending on the total intensity range covered in that frame. The gray scale is the same for all the derivative panels. Notice how many bursts are seen very clearly on the derivative panel but are almost invisible on the intensity panel; e.g., the starting frequency of the burst at 10:18:19 U.T. appears to be much higher on the derivative panel than on the intensity panel.

Intensity and derivative plots are complementary ways of visualizing the data; their association turns out to be a powerful tool for the human eye to quickly assess the main characteristics of the data.

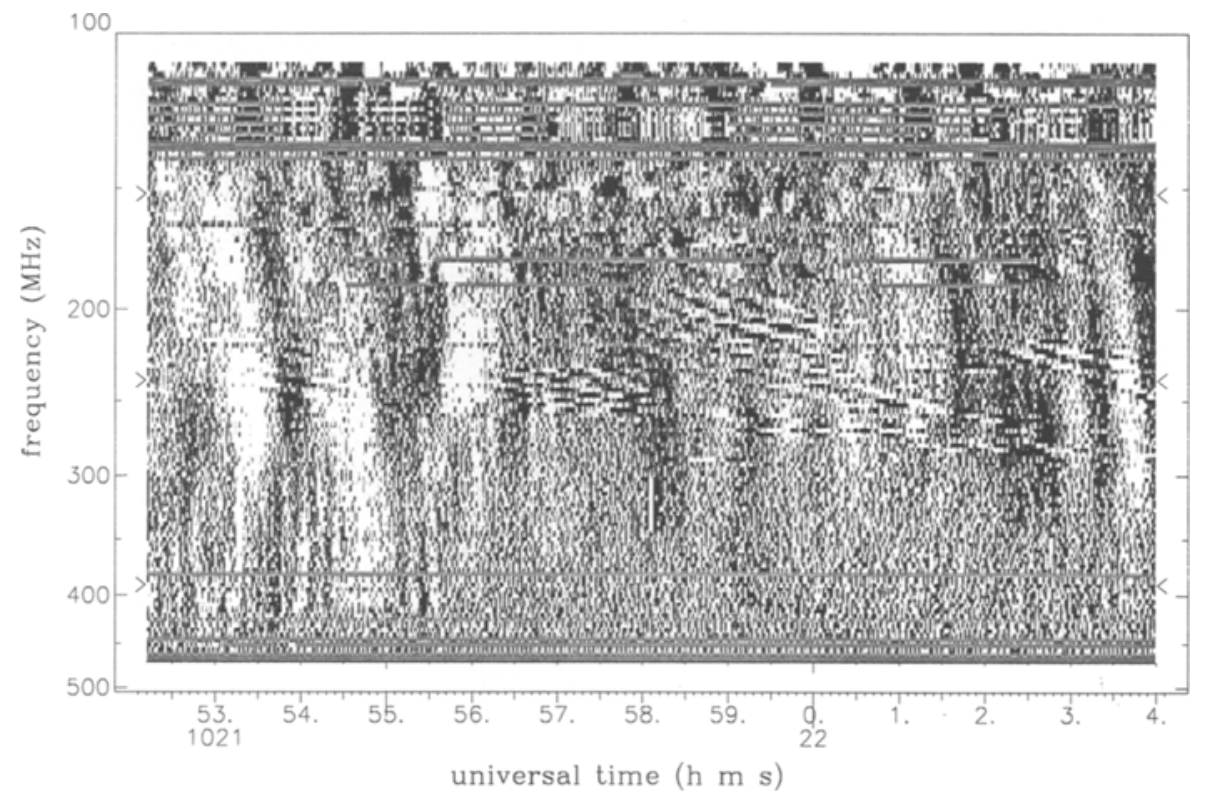

Fig. 7. Time derivative of a short excerpt of a pulsating type IV event, also showing fiber bursts. Gray-shaded scale relative to the differences between consecutive numeric values (on the same channel): black for +3 , white for -3 (see text for details). 
On the plot of Figure 6, the time resolution is limited by pixels on the paper. The intrinsic resolution of the data is much higher and requires a much more expanded scale to be fully used.

Figure 7 shows a plot at an expanded time scale which illustrates the necessity to have high resolution in time, frequency and intensity altogether. It represents the time derivative of a very small portion (12 s) of a type IV burst. We observe a complex pattern of pulsations at different time scales. The fastest (bottom left corner) have a period of the order of $0.2 \mathrm{~s}$, that is 20 times the time resolution.

Superimposed on the pulsations, we see very narrow lines in the middle of the frequency range, drifting towards higher frequencies: they are 'fiber bursts'. With an elementary bandwidth of just a few $\mathrm{MHz}$, they are at the limit of the frequency resolution of the default operating mode. The gray-level scale chosen for this plot is such that saturation levels correspond to differences between consecutive points $(0.01 \mathrm{~s}$ apart on a given channel) of +3 (black) and -3 (white) respectively in the $0-4095$ digitization scale. The intensity fluctuations observed in this example have a peak-to-peak amplitude of this order of magnitude $(\Delta I / I \approx 2 \%)$. They could not have been detected with a digitization of less than 10 bits.

At the other end of the range of time scales, Figure 8 shows type IV activity at a very contracted scale. In the center, we observe a drift at about $-20 \mathrm{MHz} \mathrm{s}^{-1}$, about $\frac{1}{10}$ of a typical type II burst. This may correspond to the slow ascending motion in the corona $\left(\approx 100 \mathrm{~km} \mathrm{~s}^{-1}\right)$ of a plasmoid producing a 'moving type IV burst' in interferometric records.

Following this event, and for about 1 hour, we observe type IV pulsations at a rate 3 orders of magnitude slower than the pulsations of Figure 7. This illustrates the need to study phenomena over a very wide range of time scales simultaneously. This plot was made with the smoothed data ( $5 \mathrm{~s}$ resolution) which have no gaps in time.

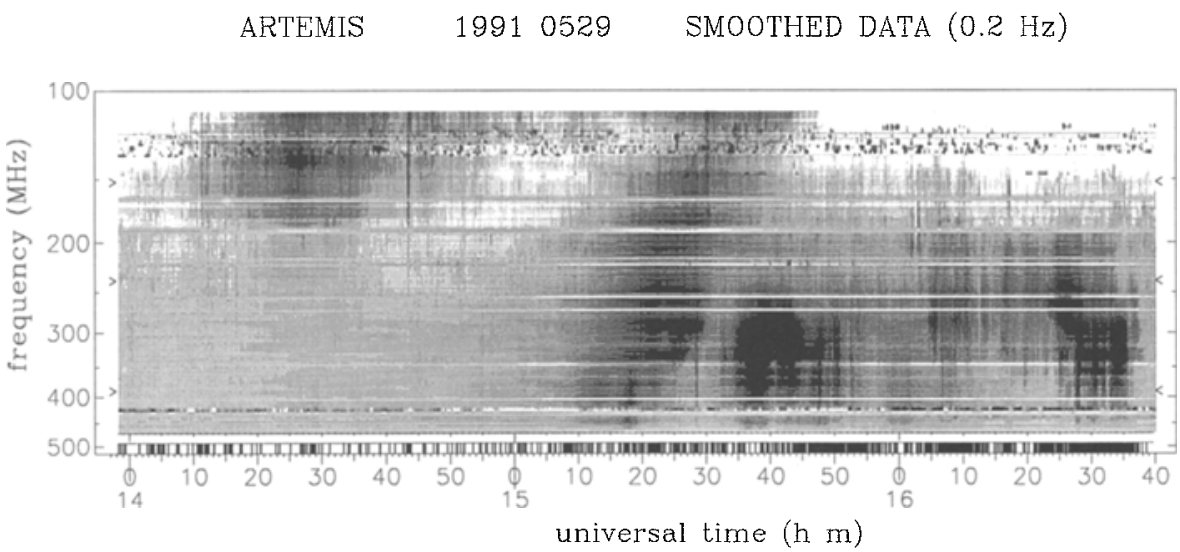

Fig. 8. Very contracted view of type IV burst (intensity). It is suggested that the drifting part (in the middle) corresponds to a 'moving type IV burst'. The black rectangles in the strip just above the time axis are the periods kept by the compression algorithm with the high time resolution ('active data' at $100 \mathrm{~Hz}$ ). (The upper line on the time axis gives minutes; the lower line gives hours.) 


\section{Conclusions and Perspectives}

The ARTEMIS digital multichannel solar radiospectrograph is characterized by its powerful real-time processing capabilities (up to 75 kbytes of raw data per second) and by its automatic functioning which eliminates most of the usual operating tasks. Since September 1989, it has been routinely recording 120 channels simultaneously at $100 \mathrm{~Hz}$, between 110 and $469 \mathrm{MHz}$. Its time, frequency, and intensity resolutions, $0.01 \mathrm{~s}, 3 \mathrm{MHz}$, and $0.01 \mathrm{~dB}$, respectively, are 10,4 , and 2 times better than with the previous system. Two other digital channels at $100 \mathrm{~Hz}$ record twenty frequencies between 30 and $80 \mathrm{MHz}$, at $5 \mathrm{~Hz}$ each. And six more digital channels at $100 \mathrm{~Hz}$ yield the twodimensional position of bursts at $75 \mathrm{MHz}$.

The data can be calibrated in real-time, so that antenna temperatures are directly stored.

We have developed and optimized a real-time data compression algorithm. Compression ratios of about 40 are currently obtained, yielding an average of about 200 Mbytes per week. Many events have been recorded with an accuracy never attained previously, some of which were presented in Section 5.

The ARTEMIS system also produces daily gray-shaded summary plots of the dynamic spectrum on A4 paper.

Systems like ours can also be used for other real-time tasks such as pattern recognition (spectral type), correlations, power spectrum analysis, etc. We have actually already tested a compression algorithm based on the FFT (Fast Fourier Transform). Its implementation on all 128 channels would require an additional specialized FFT card. Such a compression algorithm would allow us to compress the data inside active periods, without degradation of the information. Real-time pattern recognition using neural networks is also planned to be tested to adapt the compression algorithm to the type of activity.

The use of the VME bus standard and the modular architecture of our system offer a wide range of possible developments, including its utilization in connection with other receivers with very high data rates requiring sophisticated real-time processing, and its adaptability to the evolution of computer devices (mass storage on DATs or optical disks, etc.).

The data from the ARTEMIS system will be used in conjunction with several space programs related to the 'International Solar Terrestrial Physics' program including the following spacecraft: ULYSSES, GGS/WIND, SOHO, and CLUSTER. All these spacecraft, except SOHO, carry radio instruments that have been or are being developed by DESPA. We recall that the frequencies below $\approx 20 \mathrm{MHz}$ can only be observed from above the Earth's ionosphere, from a space laboratory. In particular, ARTEMIS will provice ancillary data to the WAVES investigation on the WIND spacecraft which is to be launched in 1994, allowing us to cover, for the first time, the frequency band from $8 \mathrm{~Hz}$ to $469 \mathrm{MHz}$ with common data analysis. This will provide for the first time a comprehensive description of the solar corona and heliosphere up to the Earth's orbit. 


\section{Acknowledgements}

The ARTEMIS system was funded by the 'Institut National des Sciences de l'Univers' (INSU) and was developed with a support from the Commission of European Communities (CEE) (SCIENCE program allocation granted to D.M.). We thank P. Picard, L. Denis, and G. Huntzinger for constructive discussions and their assistance for the definition and/or development of some aspects of the system. We thank G. Nicol, R. Hulin, and G. Goyon for their assistance. We particularly thank J. Renaud (Nançay Radioastronomy Station) who is running the observations and Mrs R. Crépel who runs the data exploitation at Meudon.

\section{References}

Caroubalos, C., Poquérusse, M., Bougeret, J.-L., and Crépel, R.: 1987, Astrophys. J. 319, 503.

Crépel, R., Renaud, J., Goyon, G., Bougeret, J.-L., and Poquérusse, M.: 1989, List of Archival Periods of the Solar Digital Multichannel Radiospectrograph of DESPA (1979-1988), DESPA, Observatoire de Meudon, F-92195, Meudon Cedex, France, 120 pp.

Daigne, G., Caroubalos, C., and Poquérusse, M.: 1975, L'interféromètre triangulaire utilisé pendant l'expérience Stéréo-5, DESPA 131/STER.5.

Dumas, G.: 1982, L'Onde Electrique 62, 83.

Dumas, G., Caroubalos, C., and Bougeret, J.-L.: 1982, Solar Phys. 81, 383.

Stahli, M. and Fuhrer, M.: 1987, Solar Phys. 114, 105.

Perrenoud, M. R.: 1982, Solar Phys. 81, 197.

Yagi, H.: 1928, Proc. IRE 16, 715. 